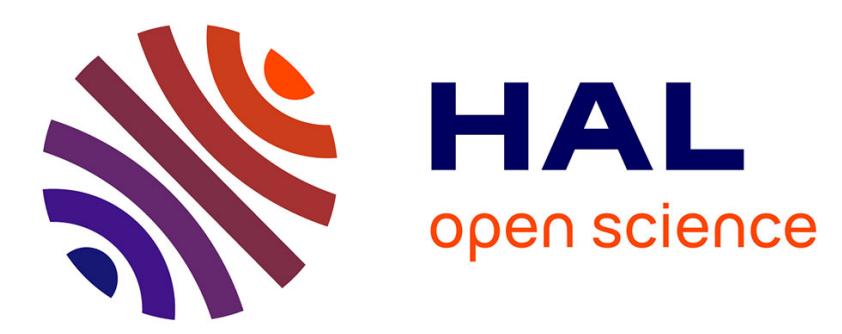

\title{
Output-based Sliding Mode Control Design for Linear Plants with Multiplicative Disturbances: the minimax approach
}

\author{
Sergiy Zhuk, Andrey Polyakov
}

\section{- To cite this version:}

Sergiy Zhuk, Andrey Polyakov. Output-based Sliding Mode Control Design for Linear Plants with Multiplicative Disturbances: the minimax approach. European Control Conference 2015, Jul 2015, Linz, Austria. hal-01160062

\section{HAL Id: hal-01160062 https://hal.inria.fr/hal-01160062}

Submitted on 4 Jun 2015

HAL is a multi-disciplinary open access archive for the deposit and dissemination of scientific research documents, whether they are published or not. The documents may come from teaching and research institutions in France or abroad, or from public or private research centers.
L'archive ouverte pluridisciplinaire HAL, est destinée au dépôt et à la diffusion de documents scientifiques de niveau recherche, publiés ou non, émanant des établissements d'enseignement et de recherche français ou étrangers, des laboratoires publics ou privés. 


\title{
Output-based Sliding Mode Control Design for Linear Plants with Multiplicative Disturbances: the minimax approach
}

\author{
Sergiy Zhuk and Andrey Polyakov
}

\begin{abstract}
In this paper we consider a problem of sliding mode control design for LTI systems with multiplicative disturbances of the input and noisy measurements of the output. First, we apply the minimax observer to provide the best possible estimate of the system's state. Then we solve a problem of optimal reaching for the observer: we design sub-optimal control algorithms generating continuous and discontinuous feedback controls that steer the observer as close as possible to a given sliding hyperplane in a finite time. The theoretical results are illustrated by an academic example.
\end{abstract}

\section{INTRODUCTION}

Sliding mode methodology has been introduced more that 50 years ago (see, for example, [19] and references therein) as the first robust control technique. A lot of research activities from purely theoretical to practical applications have been provided since then in the so-called sliding mode framework. We stress that insensitivity to the so-called matched uncertainties and disturbances is one of the main advantages of this framework [7], [20], [18].

The problem of Sliding Mode (SM) control for systems with mismatched uncertainties has been studied by many authors, e.g. [1], [15], [21]. It is recognized [6], [18] that the output-based SM control design for systems with measurement noises is a difficult problem, and hence it is important to extend the applicability of SM framework to such systems.

The paper addresses the problem of output-based sliding mode control design for a linear plant with multiplicative exogenous disturbances and bounded deterministic measurement noises. This is continuation of the research presented in [26], where the case of additive disturbances have been studied. Since the ideal sliding mode in the state space of the original system is impossible due to incomplete and noisy measurements we present a sub-optimal control law, which provides the motion of the closed-loop system as close as possible to the selected sliding surface.

The control design relies upon minimax state estimation framework [11], [4], [9] and duality argument [22], [24], [23], [25]. The minimax state estimator or observer constructs the best linear estimate of the system's state provided the uncertain parameters (model disturbance, observation error, error in the initial condition) are bounded. Statistically, this assumption corresponds to uniform distributions for

Sergiy Zhuk is with IBM Research, Server 3, Damastown Ind. Park, Dublin 15, Ireland, sergiy.zhukeie.ibm.com

Andrey Polyakov is with NON-A, INRIA - LNE, Parc Scientifique de la Haute Borne 40, avenue Halley Bat.A, Park Plaza 59650 Villeneuve d'Ascq, andrey.polyakoveinria.fr and with LAGIS UMR CNRS 8219, Ecole Centrale de Lille, BP 48, Cité Scientifique, 59651 Villeneuve-d'Ascq, France. uncertain parameters. Given the best linear estimate of the state we apply the linear separation principle and transform the problem of sliding mode control design in the state space of the original system to the optimal control problem for the observer's variables. It is worth noting that the minimax observer (in the form of a linear functional of observations), used in this paper, is optimal among all observers represented by measurable functionals of observations [13]. Thus, at least theoretically, the proposed control design can not be further improved by using observers realized by non-linear functionals of the observations. The latter agrees with the numerical comparisons of the optimal control laws designed by minimax observer and the fixed-time feedback [14] based on the second order sliding mode (2-sm) observer [5], which is nonlinear. We refer the reader to [18], [7], [20], [16] for more information about sliding mode observers.

The paper is organized as follows. The next section present the notations used in the paper. Then the problem statement and basic assumptions are considered. The minimax observed design is given in the section 4. Next the control design algorithms are discussed. Finally, the numerical simulations and conclusions are presented.

\section{NOTATION}

Through out the paper the following notations will be used:

- $\mathbb{R}_{+}=\{x \in \mathbb{R}: x>0\}, \mathbb{R}_{-}=\{x \in \mathbb{R}: x<0\}$, where $\mathbb{R}$ is the set of real number;

- $\|\cdot\|$ is the Euclidian norm in $\mathbb{R}^{n}$, i.e. $\|x\|=$ $\sqrt{x_{1}^{2}+\ldots+x_{n}^{2}}$ for $x=\left(x_{1}, \ldots, x_{n}\right)^{T} \in \mathbb{R}^{n}$;

- if $P \in \mathbb{R}^{n \times n}$ then the inequality $P>0(P \geq 0$, $P<0, P \leq 0)$ means that $P$ is symmetric and positive definite (positive semidefinite, negative definite, negative semidefinite).

- $\mathbb{L}_{\left([a, b], \mathbb{R}^{n}\right)}^{2}$ is a set of Lebesgue quadratically integrable functions $[a, b] \rightarrow \mathbb{R}^{n}$.

\section{Problem Statement}

Consider the linear output control system

$$
\begin{gathered}
\dot{x}(t)=A x(t)+(b+D g(t)) u(t), \\
y(t)=C x(t)+w(t), \\
t \in[0, T), \quad x(0)=x_{0} \in \mathbb{R}^{n},
\end{gathered}
$$

where

- $T \in R_{+}$is a finite instant of time or $T=+\infty$,

- $x \in \mathbb{R}^{n}$ is the vector of system state, 
- $u \in \mathbb{R}$ is the scalar control input,

- $y \in \mathbb{R}^{k}$ is the measured output,

- the function $g: \mathbb{R}_{+} \rightarrow \mathbb{R}^{n}$ describes multiplicative disturbances;

- the function $w: \mathbb{R}_{+} \rightarrow \mathbb{R}^{q}$ is a deterministic measurement noise,

- the system parameters $A \in \mathbb{R}^{n \times n}, b \in \mathbb{R}^{n}, C \in \mathbb{R}^{k \times n}$, $D \in \mathbb{R}^{n \times p}$ are assumed to be known and time-invariant.

We study this system under the standard assumptions ( see,[20], [7]).

Assumption 1: The pair $(A, C)$ is observable, the pair $(A, b)$ is controllable.

The noise measurements $w \in \mathbb{L}_{\left([0, T], \mathbb{R}^{q}\right)}^{2}$ and exogenous disturbances $g \in \mathbb{L}_{\left([0, T], \mathbb{R}^{p}\right)}^{2}$ are assumed to be deterministic and satisfy the inequality:

$$
x_{0}^{\top} P_{0}^{-1} x_{0}+\int_{0}^{T} w^{\top}(\tau) R w(\tau)+g^{\top}(\tau) Q g(\tau) d \tau \leq 1,
$$

where $P_{0} \in \mathbb{R}^{n \times n}, R \in \mathbb{R}^{k \times k}$ and $Q \in \mathbb{R}^{p \times p}$ are symmetric positive definite matrices. The condition (3) guarantees boundedness in $\mathbb{L}^{2}$ of the uncertain parameters $x_{0}, w, g$ of the system (1).

The admissible control law is assumed to be of $\mathbb{L}^{2}$-class, which contains both continuous and discontinuous functions.

Let $f$ be a vector from $\mathbb{R}^{n}$. In general, this paper deals with the optimal control problem

$$
\begin{gathered}
\sup _{x_{0}, w, g}\left\|f^{\top} x(T)\right\| \rightarrow \min _{u \in \mathbb{L}_{\left([0, T], \mathbb{R}^{m}\right)}^{2}} \\
\text { s.t. (1) - (3). }
\end{gathered}
$$

The optimization problem (4) appears in different control applications. On the one hand, it is a robust version of the classical optimal control problem quadratic functional in Mayer form (see, for example, [17], [3]). On the other hand, the conventional sliding mode control method (see, [20], [7]) aims at designing such a control law that realizes finite-time reaching of the given hyperplane $f^{T} x=0$ and further sliding on this plane. The sliding mode control design for the reaching phase is equivalent to (4). Indeed, obtaining a solution of this optimization problem with zero value of the functional guarantees the successful realization of the reaching phase. It is worth to emphasize that the assumption

$$
f^{T} b \neq 0
$$

is necessary for realization of the first order sliding mode control principles as well as for well-posedness of Filippov solutions for the discontinuous (sliding mode) feedback case. In this paper we make the same assumption.

Due to measurement noises and system disturbances, the sliding mode on the given surface $f^{\top} x=0$ can not be guaranteed. In this case it is important to know, which sort of feedback control should be applied in order to provide the system motion as close as possible to the mentioned surface. This paper presents an algorithm which constructes a suboptimal solution to the optimal control problem (4) in the feedback form. This algorithm relies on minimax observer design.

\section{Min-Max Optimal State Observer Design}

Let $x_{u}, x_{g}$ denote the solutions of the following ODEs:

$$
\begin{aligned}
& \frac{d x_{u}}{d t}=A x_{u}(t)+b u(t), \quad x_{u}(0)=0, \\
& \frac{d x_{g}}{d t}=A x_{g}+u(t) D g(t), \quad x_{g}(0)=x_{0} .
\end{aligned}
$$

Then, clearly, $x(t)=x_{u}(t)+x_{g}(t)$ and

$$
y_{g}(t):=y(t)-C x_{u}(t)=C x_{g}(t)+w(t) .
$$

The function $x_{g}$ may be considered as a noisy part of $x$ corresponding to disturbances from the ellipsoid (3) and $x_{u}$ represents its "mean" value corresponding to the case of zero disturbances $x_{0}=0$ and $g=0$, which forms (together with $w=0$ ) the central point of the ellipsoid (3). Since $x_{g}(t)$ does not depend on the control parameter $u$ we may first construct an estimate of the noisy part. Following [25] we introduce the following definition.

Definition 1: Assume that $l \in \mathbb{R}^{n}$ and $\widehat{U} \in L^{2}\left(0, t^{*}\right)$. A linear functional

$$
\widehat{\mathcal{U}}_{l}(y):=\int_{0}^{t^{*}} \widehat{U}^{\top}(\tau) y(\tau) d \tau
$$

is called a minimax estimate of $l^{\top} x\left(t^{*}\right)$ iff

$$
\begin{aligned}
\sigma\left(\widehat{U}, l, t^{*}\right) & :=\sup _{\left(x_{0}, g, w\right) \in \Omega^{*}}\left(l^{\top} x\left(t^{*}\right)-\widehat{\mathcal{U}}_{l}(y)\right)^{2} \\
& \leq \sigma\left(U, l, t^{*}\right), \quad \forall U \in L^{2}\left(0, t^{*}\right),
\end{aligned}
$$

where $\Omega^{*}$ is defined by (3) with $T=t^{*}$.

The number $\hat{\sigma}\left(l, t^{*}\right):=\sigma\left(\widehat{U}, l, t^{*}\right)$ is called the minimax estimation error.

In other words, the minimax estimate $\widehat{\mathcal{U}}_{l}$ has minimal estimation error $\hat{\sigma}$.

Proposition 1: Let $\hat{x}_{g}(t) \in \mathbb{R}^{n}$ be the solution of the following ODE:

$$
\left\{\begin{array}{l}
\frac{d \hat{x}_{g}}{d t}=A \hat{x}_{g}+P(t) C^{\top} R\left(y_{g}(t)-C \hat{x}_{g}\right), \\
\hat{x}_{g}(0)=0
\end{array}\right.
$$

where $P(t) \in \mathbb{R}^{n \times n}$ is the solution of the following differential Riccati equation:

$\dot{P}(t)=A P(t)+P(t) A^{\top}+u^{2}(t) D Q^{-1} D^{\top}-P(t) C^{\top} R C P(t)$, $P(0)=P_{0}$.

Then $\widehat{\mathcal{U}}_{l}\left(y_{g}\right)=l^{\top} \hat{x}_{g}\left(t^{*}\right)$ and $\hat{\sigma}\left(l, t^{*}\right)=l^{\top} P\left(t^{*}\right) l$, where $y_{g}$ is defined by (7).

We refer the reader to Appendix for the sketch of the proof.

The definition of the minimax estimate $\widehat{U}$ implies that

$$
\left(l^{\top} x_{g}\left(t^{*}\right)-l^{\top} \hat{x}_{g}\left(t^{*}\right)\right)^{2} \leq l^{\top} P\left(t^{*}\right) l .
$$

Now we recall that $x\left(t^{*}\right)=x_{u}\left(t^{*}\right)+x_{g}\left(t^{*}\right)$ and so

$$
x\left(t^{*}\right)=x_{u}\left(t^{*}\right)+\hat{x}_{g}\left(t^{*}\right)+e\left(t^{*}\right),
$$

where $e\left(t^{*}\right)=x_{g}\left(t^{*}\right)-\hat{x}_{g}\left(t^{*}\right)$ and $\left|l^{\top} e\left(t^{*}\right)\right| \leq\left(l^{\top} P\left(t^{*}\right) l\right)^{\frac{1}{2}}$. Define

$$
\hat{x}:=x_{u}+\hat{x}_{g}
$$


Then it is straightforward to check that:

$$
\left\{\begin{array}{c}
\frac{d \hat{x}}{d t}=A \hat{x}+P(t) C^{\top} R(y(t)-C \hat{x})+b u(t), \\
\hat{x}(0)=0 .
\end{array}\right.
$$

Since the calculations above hold true for any $0<t<t^{*}$, we obtain:

$$
x(t)=\hat{x}(t)+e(t),
$$

where the estimation error satisfies the inequality

$$
\left|l^{\top} e(t)\right| \leq\left(l^{\top} P(t) l\right)^{\frac{1}{2}} .
$$

The inequality (10) holds for all $l \in \mathbb{R}^{n}$ proving the following optimal (in the minimax sense) guaranteed estimate of the system state

$$
x(t) \in\left\{z \in \mathbb{R}^{n}: \quad z=\hat{x}(t)+e, \quad e^{\top} P^{-1}(t) e \leq 1\right\},
$$

i.e. the state vector $x(t)$ belongs to the ellipsoid centered at $\hat{x}(t)$ with the shape matrix $P(t)$. Recall that the formula (10) with $\forall l \in \mathbb{R}^{n}$ is just a way to define the ellipsoid (see, for example, [9]).

Remark 1: It is worth to stress that the minimax approach to observer design provides the exact estimate of the system state, namely, for any $e_{*} \in \mathbb{R}^{n}$ belonging to the estimating ellipsoid (i.e. $\left.e_{*}^{\top} P^{-1}(t) e_{*} \leq 1\right)$ and for any $t \in[0, T]$ there exist $x_{0}^{*} \in \mathbb{R}^{n}, w^{*} \in \mathbb{L}_{2}$ and $g^{*} \in \mathbb{L}_{2}$ satisfying (3) such that the equality $x(t)=\hat{x}_{u}(t)+e^{*}$ holds.

Note that $P$ depends the control parameter. This suggests to design a controller as a function of $P(t)$ and the center of the ellipsoid, that is $\hat{x}_{u}$. The next section presents the controller design.

\section{Control Design}

Let us introduce the new variable variable by

$$
\sigma=f^{\top} x
$$

Using the formula (11) we derive

$$
\sigma(T)=f^{\top} x(T)=f^{\top} \hat{x}(T)+f^{\top} e(T),
$$

where the state estimate $\hat{x}$ satisfies (9) and $e$ is the observation error.

In this paper, we are interested in obtaining a control solution, which guarantees appearance of the sliding mode on the surface $f^{\top} x=0$ at least in the space of the observer variable:

$$
f^{\top} \hat{x}(t)=0, \forall t \geq t_{0},
$$

where $t_{0} \in[0, T]$ is an arbitrary number indicating the reaching time. This assumption follows the general idea of the observer-based sliding mode control design [20], [18].

Recall that $\left|f^{T} e\right|^{2} \leq f^{\top} P(T) f$ is the best possible (in the minimax sense) estimate of the observation error. So, any control realizing sliding mode condition can be considered as a suboptimal solution of (4). Therefore, we deduce the following optimization problem

$$
f^{\top} P(T) f \rightarrow \min _{u \in \mathbb{L}_{[0, T]}^{2}, t_{0} \in[0, T]}
$$

subject to (9), (8) and (12).
Note that the task (13) is the ill-posed and difficult notlinear optimization problem, since the vector $y(t)$ is not known in advance. It just can be measured on-line at the current instant of time. So, some minimax approaches are need to be used in order to tackle this problem.

Since the observer has the zero initial condition, the reaching phase of the sliding surface can be avoided, i.e. $t_{0}=0$ in (12). In this paper, we just compare two suboptimal solutions that corresponds to the mentioned case.

\section{A. The conventional sliding mode feedback}

Following the classical methodology of the sliding mode control design [20], [7] let us define

$$
u(t)=-\left(f^{\top} b\right)^{-1} K(t) \operatorname{sign}[\hat{\sigma}(t)]
$$

where $K$ is a sufficiently large positive function .

Multiplying both sides of the system (9) by $f^{\top}$ we obtain the following equation

$$
\frac{d \hat{\sigma}}{d t}=f^{\top} A \hat{x}+f^{\top} P(t) C^{\top} R(y(t)-C \hat{x})+f^{\top} b u(t),
$$

which defines the dynamic of sliding variable $\hat{\sigma}$ for the observer state space. Substituting the representation (14) for the control law we derive

$\frac{d \hat{\sigma}}{d t}=f^{T} A \hat{x}+f^{\top} P(t) C^{\top} R(y(t)-C \hat{x})-K(t) \operatorname{sign}[\hat{\sigma}(t)]$.

Taking into account $\hat{x}(0)=0$ we derive that for any

$$
K(t)>\left|f^{\top} P(t) C^{\top} R(y(t)-C \hat{x})+f^{\top} A \hat{x}\right|
$$

the control (14) guarantees the sliding mode on the surface $f^{\top} \hat{x}=0$.

Therefore, the convectional sliding mode control is a suboptimal solution of the optimization problem (4). The formula (16) represents the rule for selection of the relay feedback gain. In practice, than the maximum magnitude of the control signal is bounded by $u_{0} \in \mathbb{R}_{+}$(i.e. $|u(t)| \leq u_{0}$ for all $t \in[0, T]$ ) the function $K$ is defined as $K(t):=$ $u_{0}\left|f^{\top} b\right|=$ const.

Evidently, the simple modification of the control law (14) as $u(t)=u_{e q}(t)+u_{d}(t)$ with

$$
\begin{aligned}
u_{e q}(t) & =-(f b)^{-1} f^{T}\left[A x(t)+P(t) C^{T} R(y(t)-C \hat{x})\right], \\
u_{d}(t) & =-(f b)^{-1} K(t) \operatorname{sign}[\hat{\sigma}(t)],
\end{aligned}
$$

also will give us one more suboptimal solution of the optimization (4) for any positive $K(t)$. Such construction follows the classical idea equivalent control-based sliding mode feedback design(see, [20]). Since the amplitude of "chattering" of the closed-loop system with the sliding mode control is usually proportional to the relay gain, it suggests that this modified sliding mode control is expected to be more reliable for practical applications.

Remark 2: Note that just one term of the Riccati equation (8) depends on the control signal $u$ so the minimization of the integral $\int_{0}^{T} u^{2}(\tau) d \tau$ will imply minimization of the term $\sqrt{f^{\top} P(T) f}$. This additionally motivates application of feedbacks with smaller magnitude of control signal . 


\section{B. The continuous control}

Evidently, the continuous control can also be designed in order to guarantee the condition (12). For instance, the continuous feedback law

$$
u_{c}(t)=-\left(f^{T} b\right)^{-1} f^{T}\left[A \hat{x}(t)+P(t) C^{T} R(y(t)-C \hat{x}(t)]\right.
$$

also ensures sliding motion of the observer state on the sliding surface $f^{T} \hat{x}=0$. Indeed, this feedback provides

$$
f^{T} \frac{d \hat{x}}{d t}=0
$$

So, taking into account $\hat{x}(0)=0$ we obtain $\hat{x}(T)=0$.

A class of continuous feedbacks can be generated by the following formula

$$
u_{\text {opt }}(t)=u_{c}(t)-\left(f^{T} b\right)^{-1} \mathrm{~h}(\hat{\sigma}(t)),
$$

where $\mathrm{h}: \mathbb{R} \rightarrow \mathbb{R}, \mathrm{h}(0)=0$ is an odd function.

Introducing the function $h$ to the feedback law may be important for practical implementation of the optimal control law in order to provide an additional robustness of the closedloop system with respect to parametric disturbances and small non-linear effects.

For example, the nonlinear function providing finite-time stabilizing control (see, for example, [2], [12]) has the form

$$
\mathrm{h}_{F T}(z)=p|z|^{\alpha} \operatorname{sign}[z]
$$

with $\alpha \in(0,1), p>0$.

The fixed-time control algorithm (see [14], [10]) can be design using the function

$$
\mathrm{h}_{F x T}(z)=\left(p_{1}|z|^{\alpha}+p_{2}|z|^{\beta}\right) \operatorname{sign}[z],
$$

where $\alpha \in(0,1), \beta>1, p_{1}>0$ and $p_{2}>0$.

In the partial cases, when $\alpha \rightarrow 1$ and $\beta \rightarrow 1$ the presented control laws become linear feedbacks.

On the one hand, the Riccati equation is depended on the control input. On the other hand, the (sub)optimal feedbacks are functions of the Riccati matrix. Therefore, existence and boundedness of the control (Riccati matrix) depended is not obvious. This paper leaves open this question for a future research.

\section{NUMERICAL SimUlations}

Consider the linearized model of the inverted pendulum of the form

$$
A=\left(\begin{array}{ll}
0 & 1 \\
1 & 0
\end{array}\right), b=\left(\begin{array}{l}
0 \\
1
\end{array}\right), D=\left(\begin{array}{l}
1 \\
0
\end{array}\right), C=\left(\begin{array}{ll}
1 & 0
\end{array}\right)
$$

and select the desired sliding surface $f^{T} x=0$ with

$$
f=\left(\begin{array}{l}
1 \\
1
\end{array}\right)
$$

Assume, in addition, that the control input is bounded as follows

$$
|u(t)| \leq 10
$$

A. Comparison of linear and sliding mode feedbacks based on minimax observer

The restrictions to energy measurement noises, exogenous disturbances and uncertainty of initial conditions is represented by the inequality (3) with the following parameters

$$
P_{0}=\frac{3 \pi^{2}}{4}\left(\begin{array}{ll}
1 & 0 \\
0 & 1
\end{array}\right), \quad Q=R=40 / 3, \quad T=10 .
$$

We compare the linear continuous feedback

$$
u_{l i n}(t)=u_{c}(t)-\left(f^{T} b\right)^{-1} \hat{\sigma}(t)
$$

with the sliding mode control of the form (14) with $K(t)=$ 10 .

The initial condition selected for simulation is

$$
x_{0}=\left(\begin{array}{ll}
0 & \frac{\pi}{2}
\end{array}\right)^{T} .
$$

The deterministic noise and disturbance functions are defined for simulations as follows

$$
w(t)=0.05 \operatorname{sign}[\sin (10 t)]
$$

and

$$
g(t)=0.05 \operatorname{sign}[\cos (10 t)]
$$

The numerical simulations has been made using explicit Euler method with fixed step size $h=0.01$.

The figures 1-8 presents the simulation results (please see the last page of the paper). It can be easily observed that the purely relay sliding mode algorithm designed using maximum admissible magnitude of the control signal has poor quality comparing with a simple linear feedback. This fact additionally confirms the drawback of large gains in the sliding mode control. Usually, it was motivated by chattering phenomenon. In this paper it is shown that, such a drawback is also related to optimality of the state estimation (see, Remark 2).

Note that the modified sliding mode control (17) with $K(t)=0.1$ has the same quality as linear control. We do not present the simulation results for this case, since the obtained figures are almost identical to the figures 1-3.

The finite-time and fixed-time algorithms provided the results similar to linear control.

\section{CONCLUSION}

The problem of the optimal reaching (as close as possible) of the selected sliding surface for the linear time-invariant disturbed system with the noised measurements of the output and with multiplicative disturbances of the input is studied using minimax observation approach. It was discovered, that the relay sliding mode control is not optimal solution to the considered problem, since estimation precision is depended on the magnitude of the input in this case. The suboptimal linear solution is presented. The performance of the designed control laws are compared on numerical simulations. 


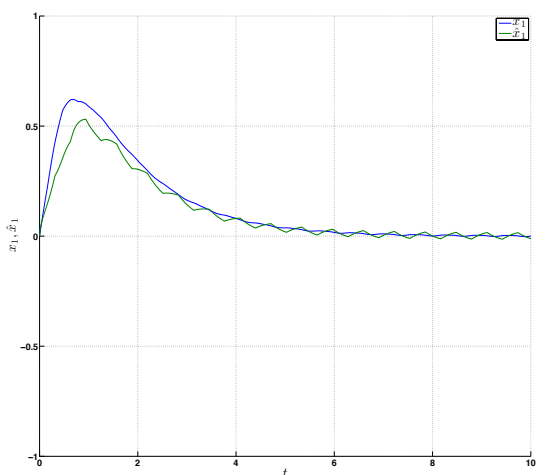

Fig. 1. The real $x_{1}$ and estimated $\hat{x}_{1}$ states for linear control $u_{\text {lin }}$.

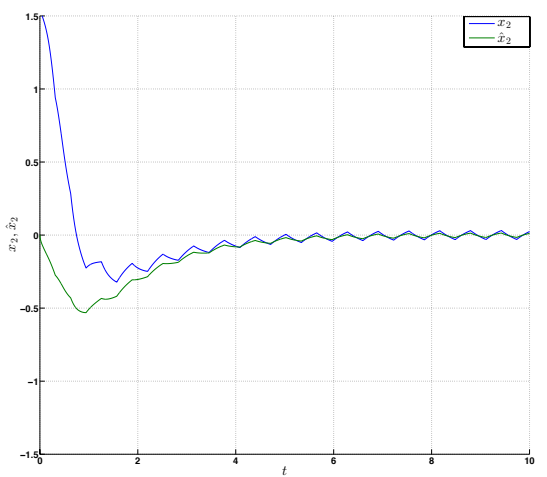

Fig. 2. The real $x_{2}$ and estimated $\hat{x}_{2}$ states for linear control $u_{l i n}$.

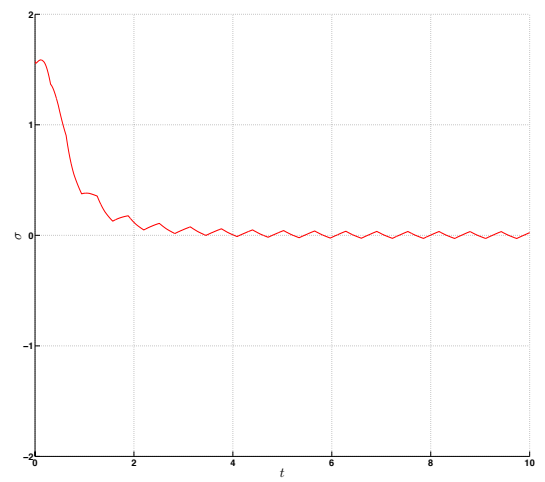

Fig. 3. The real sliding variable $\sigma$.

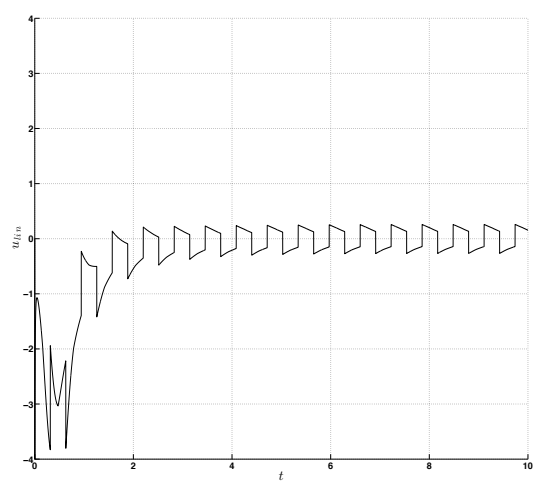

Fig. 4. The linear control input.

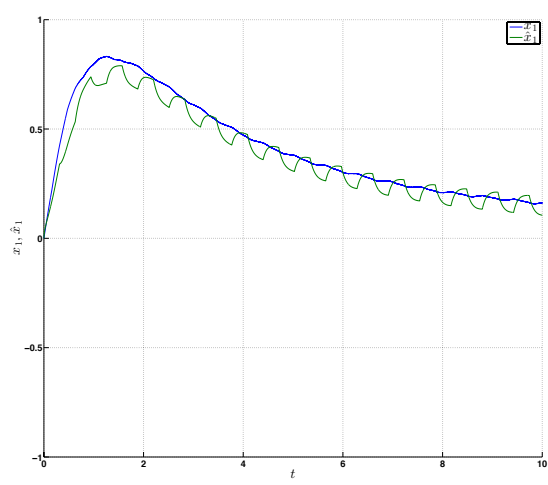

Fig. 5. The real $x_{1}$ and estimated $\hat{x}_{1}$ states for sliding mode control $u_{s m}$.

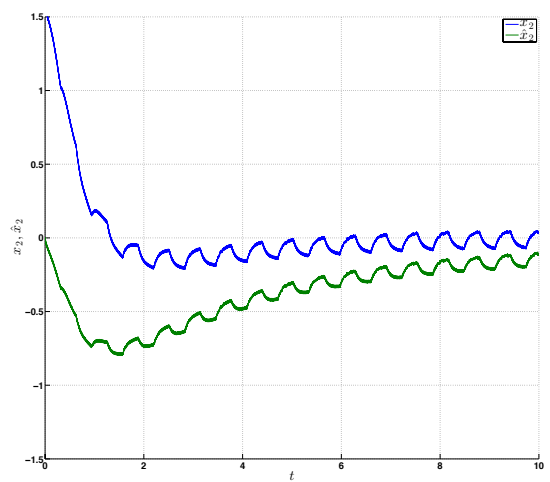

Fig. 6. The real $x_{2}$ and estimated $\hat{x}_{2}$ states for sliding mode control $u_{s m}$.

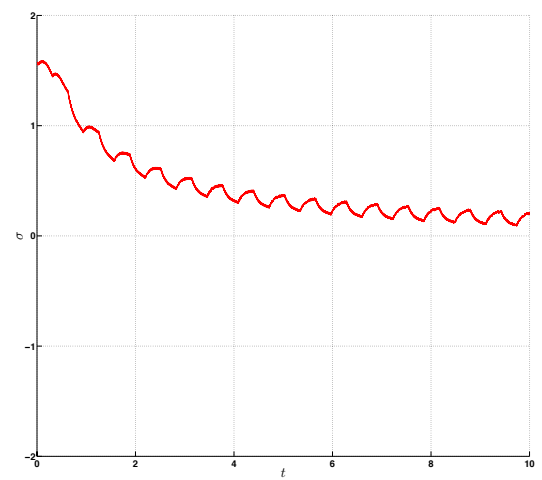

Fig. 7. The real sliding variable $\sigma$.

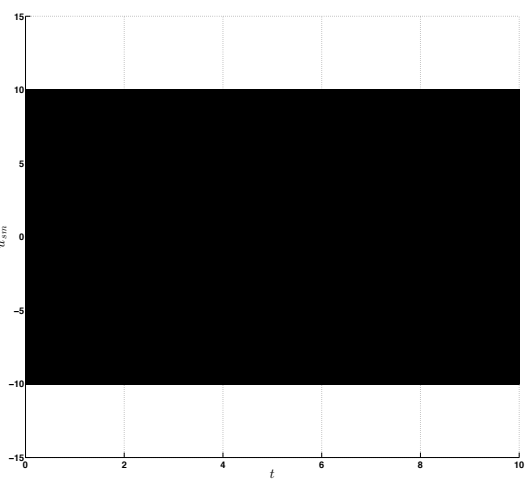

Fig. 8. The sliding mode control input. 


\section{APPENDIX}

The sketch of proof of Proposition 1.

The proof is based on the Kalman duality principle (see, [8]), which allows to prove that the minimax estimate of $l^{T} x_{g}\left(t^{*}\right)$ may be computed by introducing a linear quadratic dual control problem. Indeed, the error function admits the following representation:

$$
\begin{aligned}
& l^{\top} x_{g}\left(t^{*}\right)-\int_{0}^{t^{*}} U^{\top}(\tau) y_{g}(\tau)= \\
& z^{\top}(0) x_{0}+\int_{0}^{t^{*}}\left(\begin{array}{c}
z(\tau) \\
U(\tau)
\end{array}\right)^{\top}\left(\begin{array}{cc}
u(\tau) D & 0 \\
0 & -I_{k}
\end{array}\right)\left(\begin{array}{c}
g(\tau) \\
w(\tau)
\end{array}\right) d \tau,
\end{aligned}
$$

where

$$
\frac{d z(t)}{d t}=-A^{\top} z(t)+C^{\top} U(t), \quad z(T)=l .
$$

In this case, we have

$$
\begin{aligned}
I(U(\cdot)) & =\sup _{x_{0}, g(\cdot), w(\cdot)}\left(l^{\top} x\left(t^{*}\right)-\int_{0}^{t^{*}} U^{\top}(\tau) y_{g}(\tau)\right)^{2}= \\
& =z^{\top}(0) P_{0} z(0)+\int_{0}^{t^{*}} U^{\top}(\tau) R^{-1} U(\tau) d \tau \\
& +\int_{0}^{t^{*}} z^{\top}(\tau)\left(u^{2}(\tau) D Q^{-1} D^{\top}\right) z(\tau) d \tau .
\end{aligned}
$$

In fact, the cost $I(U)$ represents the worst-case estimation error $\sigma(U, l, T)$ (see Definition 1). It is quite natural then to minimize $I$ subject to (23) in order to find $\widehat{U}$ which has the minimal worst-case estimation error, so the minimax error $\sigma(\widehat{U}, l, T)$.

Now, using classical results in linear quadratic optimal control one finds the representation for $\widehat{U}$ in feed-back form $\widehat{U}(t)=R C P(t) z(t)$ and the corresponding Riccati equation:

$$
\dot{P}(t)=P(t) A^{\top}+A P(t)+u^{2}(t) D Q^{-1} D^{\top}-P(t) C^{\top} R C P(t),
$$

which is equivalent to (8). The observer in this case has the form (9).

The latter representation is thethat:

$$
\widehat{\mathcal{U}}_{l}(y):=\int_{0}^{t^{*}} \widehat{U}(\tau)^{\top} y_{g}(\tau) d \tau=l^{\top} \hat{x}_{g}\left(t^{*}\right)
$$

and $\hat{\sigma}\left(l, t^{*}\right)=l^{T} P\left(t^{*}\right) l$, where $x_{g}$ is defined by (7).

\section{REFERENCES}

[1] J. M. Andrade-Da Silva, C. Edwards, and S. K. Spurgeon. Slidingmode output-feedback control based on lmis for plants with mismatched uncertainties. IEEE Transaction on Industrial Electronics, 56(9):3675-3683, 2009.

[2] S.P. Bhat and D.S. Bernstein. Finite-time stability of continuous autonomous systems. SIAM Journal of Control and Optimization, 38(3):751-766, 2000.

[3] V. G. Boltyanski and A. Poznyak. The Robust Maximum Principle: Theory and Applications. Birkhauser Basel, 2012.

[4] F.L. Chernousko. State Estimation for Dynamic Systems. Boca Raton, FL: CRC, 1994
[5] J. Davila, L. Fridman, and Levant A. Second-order sliding-mode observer for mechanical systems. IEEE Transactions on Automatic Control, 50(11):1785-1789, 2005.

[6] C. Edwards, A. Akoachere, and S. K. Spurgeon. Sliding-mode output feedback controller design using linear matrix inequalities. IEEE Transactions on Automatic Control, 46(1):115-119, 2001.

[7] C. Edwards and S. Spurgeon. Sliding Mode Control: Theory And Applications. CRC Press., 1998.

[8] R. E. Kalman. On the general theory of control systems. In Proccedings of the First Congress of Automatic Control. Moscow, USSR, 1960.

[9] A. Kurzhanski and I. Valyi. Ellipsoidal calculus for estimation and control. Birkhauser Boston Inc., Boston, MA, 1997.

[10] A. Levant. On fixed and finite time stability in sliding mode control. In IEEE 52nd Conference on Decision and Control, pages 4260-4265, 2013.

[11] M. Milanese and R. Tempo. Optimal algorithms theory for robust estimation and prediction. IEEE Transactions on Automatic Control, 30(8):730-738, 1985.

[12] E. Moulay and W. Perruquetti. Finite time stability and stabilization of a class of continuous systems. Journal of Mathematical Analysis and Application, 323(2):1430-1443, 2006.

[13] A. Nakonechny. A minimax estimate for functionals of the solutions of operator equations. Arch. Math. (Brno), 14(1):55-59, 1978.

[14] A. Polyakov. Nonlinear feedback design for fixed-time stabilization of linear control systems. IEEE Transactions on Automatic Control, 57(8):2106-2110, 2012.

[15] A. Polyakov and A. Poznyak. Invariant ellipsoid method for minimization of unmatched disturbance effects in sliding mode control. Automatica, 47(7):1450-1454, 2011.

[16] A.S. Poznyak. Variable Structure Systems: from Principles to Implementation, volume 66 of IEE Control Series, chapter Deterministic output noise effects in sliding mode observation, pages $45-78$. The IET, London, UK, 2004.

[17] A.S. Poznyak. Advanced Mathematical Tools for Automatic Control Engineers: Deterministic Techniques. Elsevier, 2008.

[18] Y. Shtessel, C. Edwards, L. Fridman, and A. Levant. Sliding Mode Control and Observation. Birkhauser, 2014.

[19] V.I. Utkin. Variable structure systems with sliding modes. IEEE Transactions on Automatic Control, 22(2):212 - 222, 1977.

[20] V.I. Utkin, J. Guldner, and J. Shi. Sliding Mode Control in ElectroMechanical Systems. CRC Press., 2009.

[21] J. Yang, S. Li, and X. Yu. Sliding-mode control for systems with mismatched uncertainties via a disturbance observer. IEEE Transactions on Automatic Control, 60(1):160-169, 2013.

[22] S. Zhuk. Estimation of the states of a dynamical system described by linear equations with unknown parameters. Ukrainian Math. J., 61(2):214-235, 2009

[23] S. Zhuk. Minimax state estimation for linear stationary differentialalgebraic equations. In Proc. of 16th IFAC Symposium on System Identification, 2012.

[24] S. Zhuk. Kalman duality principle for a class of ill-posed minimax control problems with linear differential-algebraic constraints. Applied Mathematics and Optimisation, 68(2):289-309, 2013.

[25] S. Zhuk and M. Petreczky. Infinite horizon control and minimax observer design for linear daes. In Proc. 52nd IEEE Conference on Decision and Control, 2013.

[26] S. Zhuk and A. Polyakov. On output-based sliding mode control design using minimax observer. In Proc. IEEE Workshop on Variable Structure Systems, 2014. 